\title{
Reflexiones interacadémicas
}

\section{sobre el proceso de atención de enfermería}

Mtra. Rosa María Ostiguín Meléndez y Mtro. Alfredo Bermúdez González***

* Profesor Titular A de TC. Adscrita a la Unidad de Investigación y Posgrado ENEO-UNAM, línea: Enseñanza clínica.

** Profesor adscrito a la Unidad de Investigación y Posgrado de la ENEO -UNAM, línea: Filosofía e Historia del Cuidado.

El desarrollo de actividades interacadémicas en espacios de investigación sin lugar a dudas reporta la posibilidad de un intercambio de visiones sobre la disciplina y una experiencia en pro de la cultura de la Inter-multidisciplina.

En el marco de la Unidad de Investigación la ENEO-UNAM, ha instituido los jueves académicos, escenario donde se diserta sobre temas relevantes para el cuidado a la salud, tal fue el caso del Proceso de Atención de Enfermería (PAE).

En una jornada de cuatro sesiones se abordó por representantes de las diversas Academias de Enfermería e Investigadores en el tema, los diferentes escenarios en los que se evidencia el proceso, así como la problemática y forma de aproximarse al PAE.

De dicha actividad se reconocieron tres aspectos nodales en torno al PAE a saber: Aspectos semánticos-nominales, los Teóricos conceptuales y los Instrumentales; todos de gran trascendencia e impostergable abordaje.

Lo anterior inserto en una visión filosófica sobre el cuidado a partir de la aprehensión de persona y naturaleza, que lleven a considerar el verdadero humanismo con que se ha investido a la profesión de enfermería.

Palabras clave: Proceso de Atención, Proceso de enfermería, reflexiones del Proceso de atención.

\section{Abstract}

The development of interacademic activities in investigation spaces without doubt reports the possibility of an interchange of visions on the discipline and a experience for the culture of the Inter-discipline. Within the framework of the Unit of Investigation the ENEO-UNAM, has instituted academic thursdays, scene where dissert on excellent subjects for the care to the health, so was the case of the Nursing Process Attention (PAE by abbreviations in for Spanish). In cycle of four sessions one approached by representatives of the diverse Academies of Nursing and Investigators in the subject, the different scenes in which the process is demonstrated, as well at the problematic and forms to come near to the PAE. We're acknowledge of this activity, three aspects around the PAE : Aspects semantic-nominal, the conceptual theoreticians and Instrumentation; all of great importance and urgent boarding. All inserted in a philosophical vision on the care from the apprehension of person and the nature, who take to consider the true humanist whereupon she has been invested to the Nursing profession.

Key words: Process of care, nursing process, reflections attention process. 


\section{INTRODUCCIÓN}

Siempre los finales de algún ciclo auguran cambios a las vísperas temporales y con más razón si los escenarios están modificándose, así la Escuela Nacional de Enfermería y Obstetricia ve culminada en la primera gestión del Lic. Severino Rubio Domínguez la construcción de la Unidad de Investigación misma que alberga a un equipo multidisciplinario que explora desde diversos ángulos el cuidado y con ello incursiona en el saber enfermero.

Dentro de las múltiples actividades se planificó un día de diálogo que permitiera emular a los filósofos de la antigüedad, tal vez no se haga en un pórtico o en un campo como la historia lo refiere y sí dentro de una aula, que sin duda generará conocimiento que fortalecerá a la disciplina, a los proyectos y a la docencia misma.

Se ha caminado por los pasillos de la ciencia con conocimientos prestados, sostenidos a pensamientos, usos y costumbres diferentes a los de la sociedad mexicana a la cual se pertenece y en la que se enseña la disciplina; confundiendo el conocer por el hacer repetido, escenario donde se ha abrigado el Proceso Atención de Enfermería (PAE).

Así en la Unidad, se propuso coordinar una actividad denominada "jueves académicos" en donde semanalmente expositores varios presentan a la comunidad en general temáticas de interés que permiten tanto un intercambio de ideas como ir creando una "cultura de discusión interprofesional".

De este modo uno de los primeros encuentros de trabajo de este tipo, se generó en torno al PAE.

A partir de reconocer al PAE como un punto de convergencia para varios proyectos de investigación y planes de estudio en pregrado y posgrado, se llevó a cabo la jornada de disertación sobre el mismo (26 de abril, 3, 17 y 24 de mayo del 2007). Todo ello con representación de las academias de enfermería fundamental, del adolescenteadulto-anciano y del niño; además de una última sesión donde se expuso una experiencia investigativa en el terreno de estrategias para fortalecer la enseñanza del PAE.

En este marco, el presente documento expone una serie de reflexiones que fueron vertidas en ese espacio académico y no pretende más que ser una evidencia de los señalamientos realizados, además de constituirse en una forma de reconocer y patentizar áreas de oportunidad y experiencias cuando la academia confluye.
Empezar a considerar las situaciones que hay entorno al PAE es una oportunidad de reflexionar en diversos aspectos que durante la docencia de la enfermería todos y cada uno hemos experimentado de alguna u otra forma, sin embargo el fenómeno tiene múltiples aristas que en la medida que se exploren ofrecerán elementos para su desarrollo y con ello consensos para útiles y necesarios para la docencia en el cuidado.

\section{Problemas reconocidos entorno al PAE}

El "problema" de abordar el PAE podría dividirlo en tres aspectos sobre los cuales giraron las disertaciones: 1) los que se relacionan con el terreno semántico-nominal atribuido al PAE; 2) los teórico-conceptuales y 3) los instrumentales.

\section{Aspecto semántico-nominal del PAE}

Uno de los puntos sobre los cuales se inició la reflexión fue justamente el aspecto semántico-nominal del Proceso de Atención de Enfermería, lo mismo se llamó proceso enfermero, método enfermero o proceso de cuidado; probablemente estos términos no reflejan más que usos o interpretaciones del PAE en el tiempo, algunos aluden a dos momentos: el primero situado en la década de los 70's como parte de una forma de "enseñar" procedimientos en enfermería y el segundo usado a mediados de la década de los 90's cuando se introducen textos españoles que inician un planteamiento del PAE como Método, sin ir mas haya de esto, sería de utilidad contextuar desde una investigación histórica su uso.

La multiplicidad de formas de aludir al PAE; condujo a la consideración de las implicaciones que se le atribuyen lo mismo como técnica didáctica, técnica para hacer procedimientos, estrategia del proceso didáctico-pedagógico de la enseñanza de la enfermería y como recurso metodológico para la construcción de la disciplina. Es probable que esto de paso a una visión poco clara en torno a que si el PAE es una construcción epistemológica, una "técnica" para actuar o ambas; todas dignas de ser estudiadas.

\section{Aspectos teórico-conceptuales}

En lo referente a los aspectos teórico-conceptuales, probablemente los de fondo, se señaló que existe una urgencia de contextuar al PAE en el cuidado, algunos intentos parciales de parte de las academias se han intentado desde el metaparadigma, otros desde una escuela del pensamiento enfermero y hasta desde un enfoque holístico, que se identifica poco claro ya que implica una reflexión filosófica profunda sobre el término, misma que aun no se ha iniciado. 
De la mano a lo anterior está la necesidad de iniciar trabajos que aborden el concepto de persona/hombre que se tiene en la disciplina, ya que a partir de éste referente, se desarrollarán estrategias para aproximarse a él y ofrecer cuidado; labor por supuesto titánica, pero de impostergable abordaje.

Reflexionar sobre el marco teórico que debe sustentar al PAE, conlleva a reconocer la necesidad de un argumento ético-filosófico, que permita rebasar el carácter dogmático que se le ha atribuido, en donde se asume como una sentencia corta y sucinta del "proceder en la enfermería" y esto conduce a una reflexión en el sentido humanista del $P A E$, es decir explorar en el pensamiento ontológico que nos acerque a la naturaleza del hombre y su abanico de posibilidades cuando se coloca al frente de aquel semejante que le demanda atención ante la ausencia de la autonomía integral como sujeto interactivo.

Paralelamente a lo anterior se reconoció que el PAE es un elemento que se ha desarrollado en un escenario positivista que le ha dado un significado utilitarista y fragmentario que se corrobora o legitima hoy por hoy en la realidad de las instituciones de salud, en donde el hombre se descompone en sus "elementos". Revertir este esquema es sin duda un ejercicio profundo de la enfermería, en donde es necesario partir de una visión integral de la persona y no de un proceso de "reconstrucción" de la misma, que alude finalmente a la suma de partes o piezas que lejos esta de la integralidad.

Para que se construya la disciplina se reconoce la urgencia de una visión fenomenológica, aunque existen otras corrientes como la de las mentalidades que brinda un enfoque que permita retomar el escenario o contexto, que de pertinencia a las acciones de cuidado señaladas para una persona como sujeto o como colectivo.

\section{Aspectos instrumentales}

Desde el punto de vista instrumental, el PAE tiene diversas áreas de oportunidad; probablemente una de las más sobresalientes y reiteradas es la limitada habilidad para la valoración clínica, que de no ser adquirida paulatina y consistentemente, coarta seriamente el desarrollo del PAE y se constituye en un elemento restrictivo para aproximarse al sujeto de cuidado.

Sin duda de lo anterior se desprenden los intentos por construir instrumentos de valoración atendiendo a propuestas filosóficas o modelos de enfermería (escuela de las necesidades, por lo referido) lo que en ocasiones lleva implícito el riesgo de ocuparse más de la forma misma que del conocimiento del sujeto de cuidado.

Esto probablemente sea un preludio de las inquietudes vertidas en el sentido de que se ha ubicado al PAE como una actividad al margen del diagnóstico médico, perdiendo de vista a la persona y centrándose en la enfermedad.

Desde el punto de vista instrumental queda claro que "aprender" el PAE no significa dominio del mismo y que es preciso reflexionar sobre los alcances que paulatinamente el alumno tendrá que desarrollar durante su formación, siendo una estrategia el pensamiento critico.

El pensamiento crítico es un recurso para el PAE o una táctica para enseñar la metodología, siempre y cuando sea visto como un proceso didáctico pedagógico y un fenómeno social, que apunte al desarrollo del juicio clínico y la toma de decisiones en el campo del cuidado de la salud de las personas y con ello rebasar la visión tecnificada de su uso, a través de la solución creativa de problemas que pueden desencadenarse por medio de estrategias como el uso didáctico de la pregunta o el análisis de casos.

En este terreno de incertidumbre conceptual y contextual del PAE sin duda se explica esa visión dogmática con que se ha asumido y enseñado, lo que implica reflexionar en cuestionamientos como los siguientes: ¿es el PAE la razón de ser de la enfermería? o ¿es un recurso para el despliegue del cuidado?, sea cual fuere la respuesta reconocerse en alguna postura explica una forma de aplicarlo y enseñarlo.

A manera de conclusión, parecería que hablar del PAE implica una consideración colegiada mas haya del como se entiende, lo que conlleva sin duda una meditación en el terreno epistemológico de la disciplina.

Por otro lado es necesario valorar la posibilidad de establecer experiencias fundamentadas con una visión más fenomenológica del cuidado en donde el PAE recupere en ese marco al todo de la persona sin fragmentarlo.

El concepto de cuidado determina el propio del PAE y por ende de su forma de interpretarlo y usarlo, así que existe una necesidad de realizar estudios sobre el asunto.

Por último en el discurso docente queda claro que hay estrategias como el desarrollo de experiencias que posibilitan y alientan el pensamiento critico para hacer del PAE un recurso que impulse la disciplina de enfermería, pero que haga visible el cuidado ante las personas. 
Sin duda las experiencias de disertación entre docentes interesados en una temática y dentro de un espacio de discusión académico como el que se da en la Unidad de investigación de la ENEO ofrece a todos y cada uno de los participantes la posibilidad de iniciar, continuar o desarrollar grupos de trabajo, de discusión o de investigación sobre puntos de interés común y de gran trascendencia como sin duda lo es el PAE.

Finalmente sistematizar lo vertido en estos foros permite mostrar un mosaico de visiones al respecto de un problema concreto y con ello múltiples formas de abordaje.

Agradecemos a todos y cada uno de los asistentes y participantes por sus opiniones, en particular a los representantes de academia:

Mtra. Sandra Sotomayor.- Academia de Enfermería Fundamental.

Lic. Aurora Benavides.- Academia de Enfermería del Niño.

LEO/EEC. Querubín Enriquez.- Academia del Adolescente, Adulto y Anciano.

Mtra. Laura Morán Peña. Prof. Titular de TC en ENEO.

Mtra. Zoila León Moreno.- Integrante del Proyecto PAPIME: EN223403. Tutoría clínica de enfermería para el fortalecimiento del aprendizaje de los alumnos en la práctica hospitalaria

\section{REFERENCIAS BIBLIOGRÁFICAS}

Armar, A;Videla,N; Torre, M. Tendencias y perspectivas de la ciencia, Enfermeria Global- Esp- 2006, nov, 9.

León Román, CA. Enfermería Ciencia y arte del cuidado, Rev. Cubana de Enfermería, 2006, oct-dic, 22 (4)

Scherer, Seine, Alves, Paires; Scherer, Edson Arthur. Reflexiones sobre la enseñanza de la enfermería en la pos modernidad, metáfora de una laguna teórico-práctica. Revista Latina - am- Enfermagen, Brasil, 2007, mayo-junio, 15 (3): 498501

Vargas Chávez, MY. Algunos fundamentos técnicos para el cuidado de enfermería. Rev. Mexicana de enfermería cardiológica, 2007, enero, 1 (1): 28-30

Watson, Jean. La teoría del cuidado humano de Watson y las experiencias subjetivas de vida: factores caritativos/caritas, texto Contexto, Enfermer, 2007, enero-marzo, (1): 129-135.

\section{DIRECCIONES DE CORRESPONDENCIA}

Mtra. Rosa María Ostiguín Meléndez: rosyostiguin@hotmail.com Mtro. Alfredo Bermúdez González: hereje@prodigy.net.mx

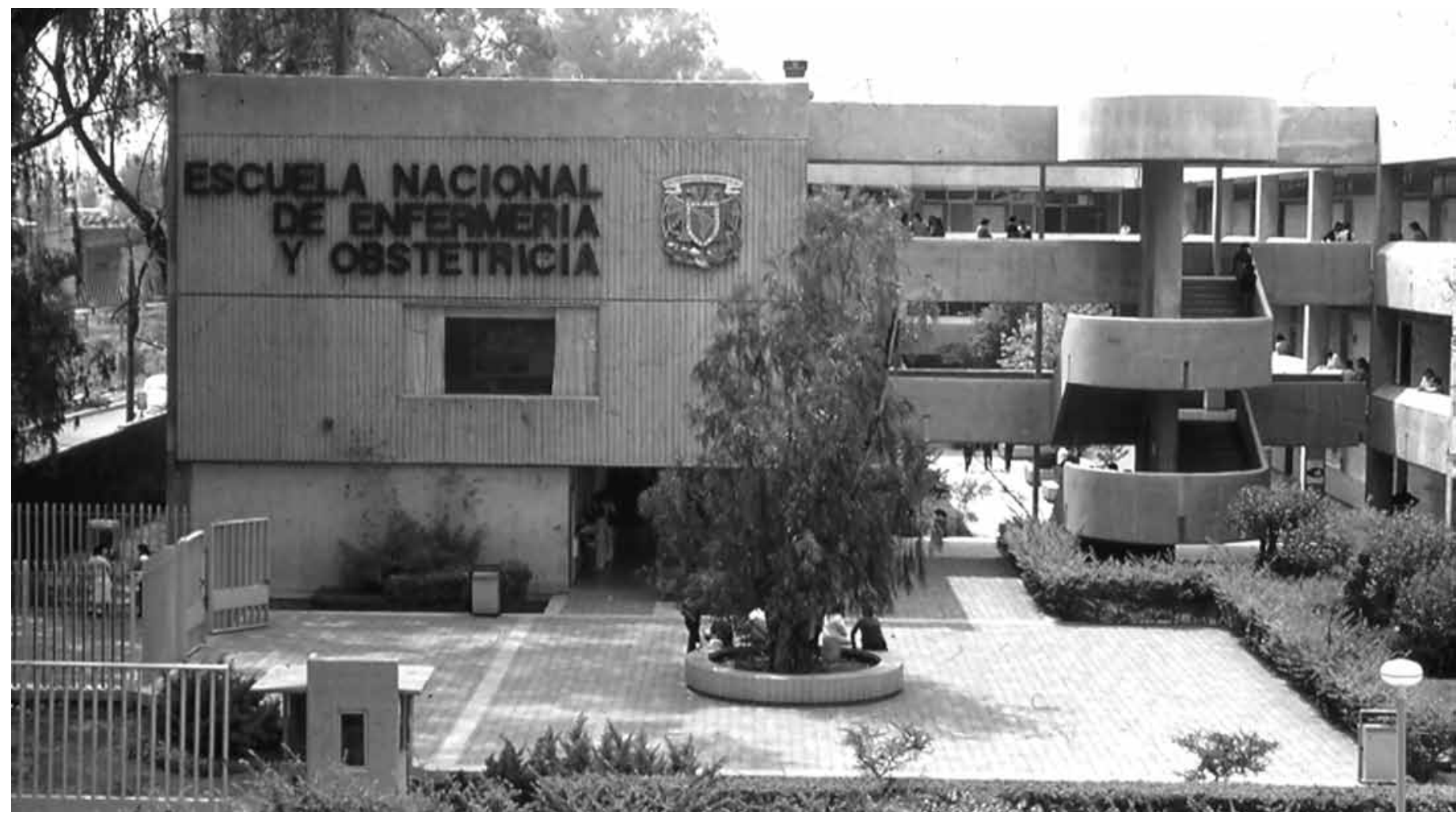

